\title{
A 7-year-old girl with progressive gait disturbance
}

\begin{abstract}
Pantothenate kinase-associated Neurodegeneration (PKAN) is the most common disorder of the Neurodegeneration with brain iron accumulation (NBIA) group. The common presentations are dystonia, Parkinsonism, dysarthria, gait disturbance, dementia and retinal degeneration. In this paper we present a 7-year old Girl that present with progressive gait disturbance in a non-consanguineous marriage.
\end{abstract}

Keywords: PKAN disease, NBIA, Gait disturbance, spasticity, dysarthria
Volume I Issue 5 - 2017

\author{
Saeed Razmeh \\ Iran University of Medical Sciences, Iran
}

Correspondence: Saeed Razmeh, Iran University of Medical Sciences, Tehran, Rasoul Akram Hospital, Iran, Tel 98937759 4424, Email srazme82@gmail.com

Received: August 02, 2017| Published: August 18, 2017

\section{Introduction}

Neurodegeneration with brain iron accumulation (NBIA) is a heterogeneous group of inherited neurodegenerative diseases, characterized predominantly by a progressive abnormal movement due to iron deposition in the brain, especially in the basal ganglia, and less common in the substantia nigra and adjacent areas. ${ }^{1,2}$ PKAN is an autosomal recessive disorder characterized by mutations in the gene encoding a mitochondrial pantothenate kinase (PANK2) at locus $20 \mathrm{p} 13$. It is the most common disorder of the NBIA group. The classic clinical presentation of PKAN is characterized by early-onset and rapidly progressive course and including dystonia, spasticity, rigidity, dysarthria and dementia. ${ }^{3,4}$ We report a case of PKAN that present with progressive gait disturbance in a non-consanguineous marriage.

\section{Case presentation}

We report an 11-years-old Iranian female present from nonconsanguineous marriage, with history of normal birth and milestones, no history of seizure, with gait disturbance presented at 4 years of age, starting from lower extremity stiffness and frequent falls while walking. Her disease followed by spasticity in the upper extremity. The disease gradually progressed with dysarthria, dysphagia and drooling. Over next year, she had abnormal episodic posturing at oromandibular and then at upper limb, neck, lower limbs, and sometimes trunk follow by generalized dystonia. She had not any history of visual or cognitive disturbance. The neurologic examination revealed the presence of Parkinsonism feature including masked face, the rigidity of four limbs \& axial rigidity, oromandibular and generalized dystonia as well as the presence of hyperreflexia and spasticity of the lower limbs, with flexor plantar reflex. The neuro-ophthalmologic exam, including fundoscopy excluded retinitis pigmentosa and Kayser-Fleischer ring. The examination also revealed mild cognitive impairment with mini mental status examination (MMSE). Her laboratory workup included normal serum copper, ceruloplasmin, and ferrit in levels and no acanthocytes were found on a blood smear. Brain MRI shows bilateral symmetrical T2 weighted hypo intensity in the globus pallidus with central hyper intensity, suggestive of typical 'Eye of Tiger' sign (Figure 1). She was homozygous for PKAN2 mutation and finally, the classical PKAN was diagnosed. She is now under symptomatic treatment with Botulinum Toxin A and trihexyphenidyl
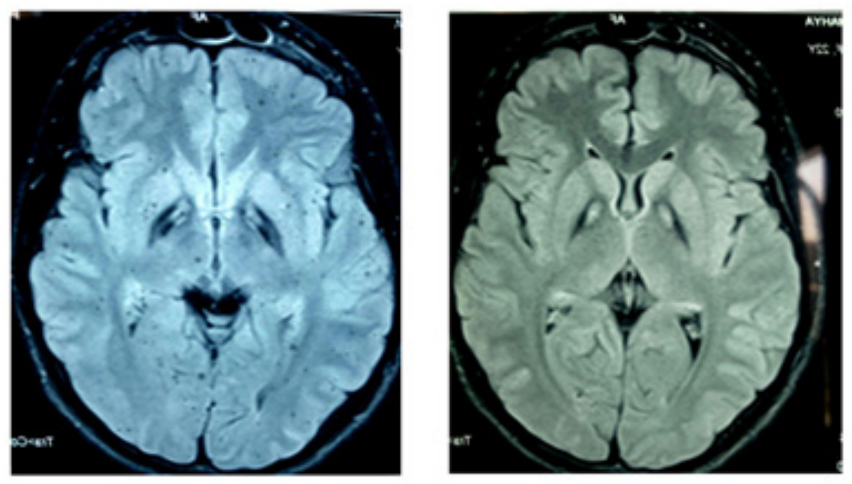

Figure I (A) Axial SWI weighted, (B) fluid attenuation inversion recovery MRI showing low signal intensity in the bilateral globus pallidus with a medial area of signal intensity, presenting the classic "eye-of-the-tiger" sign.

\section{Discussion}

There are ten forms of NBIA patients including eight with autosomal recessive inheritance, one autosomal dominant form, and one X-linked dominant inheritance. The most common types are pantothenate kinase-associated Neurodegeneration (PKAN) which are $30-50 \%$ of NBIA cases. ${ }^{3,4}$ PKAN has the classic form, with early onset and rapid evolution, and the atypical form, with later onset and slower progression. The typical clinical presentation of PKAN is early-onset (mean age is 14 years-range from 1 to $28 \mathrm{y}$ ) and rapidly progressive course. ${ }^{5}$ The affected child presents gait impairment, movement disorders particularly dystonia and Parkinsonism, spasticity and brisk tendon reflexes, dysarthria, mental changes and dementia and retinal degeneration. ${ }^{6}$ The majority of individuals $(85 \%)$ become wheelchair bound within 15 years after the beginning of symptoms. The most important MRI finding in PKAN is "eye-of-the-tiger" sign that is a bilateral hyperintense signal in the medial globus pallidus, within hypointense signal on T2-weighted scanning. ${ }^{6,7}$ The differential diagnosis of HSD includes Wilson disease, a Juvenile form of Huntington disease, ceroid lipofuscinosis, Machado-Joseph disease, Neuroacanthocytosis. The mutation in PANK2 gene cause this disease that allows iron accumulate in basal ganglia but the exact mechanisms and role of iron in this disease is unknown and in the studies that 
use from iron chelating for treatment of disease, the results was controversial ${ }^{8}$ and as yet there is no treatment for PIKAN disease and management is symptomatic therapy. ${ }^{9}$ Spasticity and stiffness reduce moderately with baclofen. The anticholinergic and dopamine agonist relieve the rigidity and The Botulinum toxin (BTX) can use for severe dystonia and drooling. ${ }^{10}$ Our case has the homozygote mutation in PIKAN2 from a non-consanguineous marriage that is the first case that reported with this features. In conclusion as previous study shows $s^{3,7,8}$ mutation spectrum in Iranian population might be wide and further research is needed to provide data on the identification of common PANK2 mutations in Iranian population. ${ }^{11}$

\section{Acknowledgements}

None.

\section{Conflict of interest}

The author declares no conflict of interest.

\section{References}

1. Limongi JC. Neurodegeneration with brain iron accumulation. Arq Neuropsiquiatr . 2016;4(7):517-518.

2. Fasano A, Shahidi G, Lang AE, et al. Basal ganglia calcification in a case of PKAN. Parkinsonism Relat Disord. 2017;36:98-99.

3. Dezfouli MA, Alavi A, Rohani M, et al. PANK2 and C19orf12 mutations are common causes ofneurodegeneration with brain iron accumulation. Mov Disord. 2013;28(2):228-232.
4. Schneider SA. Neurodegeneration with brain iron accumulation. Curr Neurol Neurosci Rep. 2016;16(1):9.

5. Salomão RP, Pedroso JL, Gama MT, et al. A diagnostic approach for neurodegeneration with brain iron accumulation: clinical features, genetics andbrain imaging. Arq Neuropsiquiatr. 2016;74(7):587-596.

6. Lee JH, Park J, Ryu HS, et al. Clinical Heterogeneity of Atypical Pantothenate Kinase-Associated Neurodegeneration in Koreans. J Mov Disord. 2016;9(1):20-27.

7. Dezfouli MA, Jaberi E, Alavi A, et al. Pantothenate kinase 2 mutation with eye-of-the-tiger sign on magnetic resonance imaging in three siblings. Iran J Neurol. 2012;11(4):155-158.

8. Ghafouri-Fard S, Yassaee VR, Rezayi A, et al. A Novel Nonsense mutation in PANK2 Gene in Two Patients with Pantothenate KinaseAssociated Neurodegeneration. Int J Mol Cell Med. 2016;5(4):255-259.

9. Diaz N. Late onset atypical pantothenate-kinase-associated neurodegeneration. Case Rep Neurol Med. 2013;2013:860201.

10. Kruer MC, Boddaert N, Schneider SA, et al. Neuroimaging features of neurodegeneration with brain iron accumulation. AJNR Am J Neuroradiol. 2012;33(3):407-414.

11. Hayflick SJ, Hartman M, Coryell J, et al. Brain MRI in neurodegeneration with brain iron accumulation with and without PANK2 mutations. AJNR Am J Neuroradiol. 2006;27(6):1230-12333. 